\section{How easily malaria can be missed}

\author{
Philip J Allan Hasan I S Tahir
}

J R Soc Med 2006;99:201-202

The diagnosis of malaria can be missed when there is a significant time lag from exposure and negative initial tests. As clinicians we must always consider the diagnosis in the appropriate clinical setting as a missed diagnosis can potentially result in serious consequences.

A 27-year-old Nigerian woman presented with a 2-day history of headaches, mild photophobia, sweats and fever. She arrived in the UK from Nigeria 8 months previously and had not been back since. On admission she was unwell with a temperature of $38.5^{\circ} \mathrm{C}$, pulse $110 / \mathrm{min}$ but otherwise haemodynamically stable. Examination revealed a soft ejection systolic murmur and signs of meningism, but her neurological assessment was otherwise entirely normal. Her initial investigations showed a haemoglobin level of $11.8 \mathrm{~g} / \mathrm{dL}$, white cell count $3.9 \times 10^{9} / \mathrm{L}$, lymphocytes $0.5 \times 10^{9} / \mathrm{L}$, platelets $118 \times 10^{9} / \mathrm{L}$, C-reactive protein $121 \mathrm{mg} / \mathrm{L}$; urea and electrolytes and liver function tests were normal.

She was commenced on ceftriaxone $2 g$ daily with a presumptive diagnosis of meningitis. The patient had a normal computerized tomograph of the head, which was followed by a normal lumbar puncture. Two sets of blood cultures and a mid-stream urine sample had no growth after $48 \mathrm{~h}$. The initial admission pyrexic thick and thin blood film and malarial antigen test (Optimal, DiaMed) were negative.

She improved initially with antibiotics and intravenous fluids. $24 \mathrm{~h}$ into her admission, having been reviewed by a number of senior doctors, her working diagnosis was changed to one of a viral illness and plans were made for her discharge. Malaria was felt to be unlikely given that not only was she 8 months post-exposure for malaria, her blood film and antigen test were negative.

However, on the day of discharge she was found to spike a temperature of $38^{\circ} \mathrm{C}$ and remained thrombocytopenic $\left(108 \times 10^{9} / \mathrm{L}\right)$. It was decided that in view of her country of origin and the fact that she remained thrombocytopenic, malaria still needed to be considered. Even though a thick and thin blood film and malarial antigen test (Optimal, DiaMed) were negative, we felt three

Emergency Medicine Centre, Whipps Cross University Hospital, London E11 1NR, UK

Correspondence to: Dr Hasan Tahir

E-mail: hasan.tahir@whippsx.nhs.uk

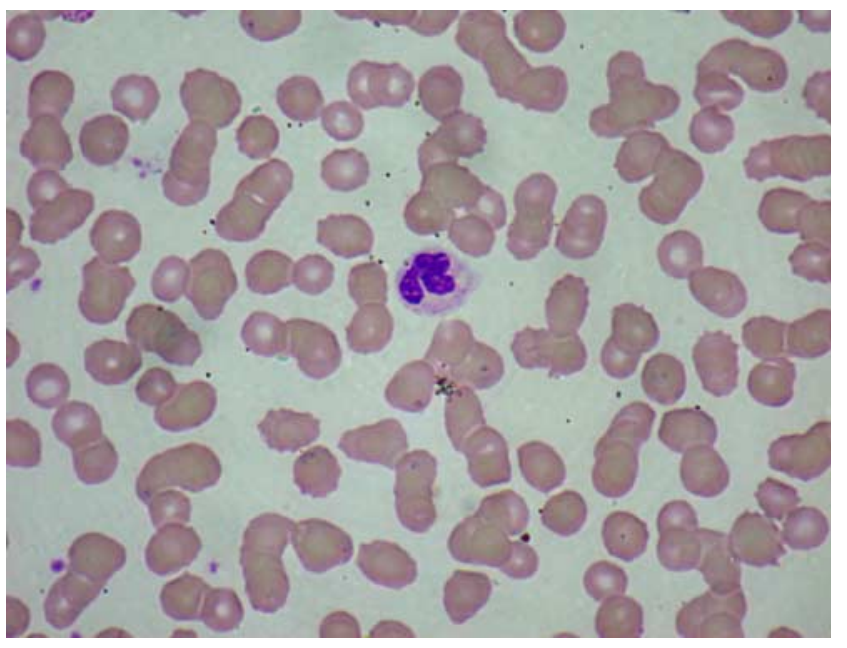

Figure 1 Patients blood film showing malarial parasites (in colour online)

negative blood films were needed before excluding malaria and labelling her as a patient with a viral illness.

Interestingly, a repeat blood film showed malarial parasitaemia of $5 \%$ Plasmodium falciparum (Figure 1). Her haemoglobin dropped to $9 \mathrm{~g} / \mathrm{dL}$ and platelets dropped to $93 \times 10^{9} / \mathrm{L}$ over the next 2 days, which then recovered. She was treated with intravenous quinine $(10 \mathrm{mg} / \mathrm{kg})$ for $48 \mathrm{~h}$ and converted to oral quinine for 7 days followed by three tablets of oral pyrimethamine with sulfadoxine (Fansidar). She was discharged home with a negative parasitaemia.

\section{COMMENT}

The Office of National Statistics reports increasing international travel with 25 million visitors to the UK and 61 million trips abroad made by UK residents in 2003. According to the Health Protection Agency there were 1722 cases of malaria in the UK in 2003, 78\% were P. falciparum, all of which were diagnosed within 5 months of arrival in the UK. Their statistics also show that $59 \%$ of malarial cases in UK are from those of African ethnicity; patients who were infected in Nigeria contribute one-third of all $P$. falciparum cases in UK and 2 out of 16 deaths in 2003.

Immunity to malaria is acquired slowly, is incomplete and on leaving the infectious environment it falls over about 1-2 years. Those who have been out of exposure for more than 1 year or were been born in UK are classed as nonimmune. These individuals, if exposed usually, develop malaria within 1 month of leaving the endemic area. In contrast those who are from endemic areas and arrive in the UK as immigrants, visitors or students (as was our patient) are classed as semi-immune. In these individuals, and those who have taken malaria prophylaxis, incubation can be significantly prolonged and may have milder symptoms. In 
those countries with high transmission rates, severe disease is usually a disease of the young (1 month to 5 years) or non-immune adults (the vast majority of travellers to endemic areas). Children in areas of high transmission often show tolerability of the parasites due to constant parasitaemia without signs of disease, and paradoxically those living in low transmission areas have high clinical disease burden. ${ }^{1}$

Reviewing the Health Protection Agency statistics, of the 1722 cases reported in 2003, 52\% were visiting family and friends in their country of origin and probably nonimmune as they had been born or lived in the UK for some time; $22 \%$ were from endemic areas (immigrants, visitors, students); and $26 \%$ were almost certainly non-immune (business travel, holiday, expatriates). The diagnosis of malaria in the $22 \%$ group, who arrive from an endemic area and are semi-immune, is often difficult because they can present late with clinical malaria (as demonstrated with our patient being in the UK for 8 months prior to presentation).

Our case was notable in that our patient had an initial negative microscopy and malaria antigen test. The latter is a new method for detecting malaria. It is a rapid dipstick immunoassay that detects circulating antigens of either histidine rich protein-2 of falciparum (HRP-2f) or plasmodium lactate dehydrogenase $(\mathrm{pLDH})$. These are near-patient testing kits that have recently been developed to ease and speed up diagnosis, particularly in places that do not have access to microscopes or trained laboratory staff. The malarial antigen test Optimal (DiaMed) used in this case detects presence of $\mathrm{pLDH}$. This assay can detect parasitaemia levels of 100-200 parasites/ $\mu \mathrm{L}$ of blood, equivalent to $0.002 \%^{2}$ and has been found to have a sensitivity of $95.3 \%$ and a specificity of $100 \%$ for P. falciparum. ${ }^{3}$ However, a meta-analysis of 10 popular brands showed that HRP-2 was more accurate than the pLDH tests. ${ }^{4}$ Though quick to use and easy to read they are not as accurate nor as good at detecting species as microscopy using thick and thin films, hence microscopy remains the method of choice.

We believe most general physicians would have felt that on initial presentation our patient was unlikely to have malaria. She presented with non-specific symptoms and signs of meningism; over 8 months had elapsed since her return from Nigeria; and the initial investigations were negative for malaria. Many might have put her presentation down to a viral illness, though she had the characteristic lymphopenia and thrombocytopenia for malaria (sensitivity for thrombocytopenia in those with malaria is $60 \%$, and specificity is $95 \%) .{ }^{5}$ However, a study in Nigeria found that headache, fever, chills and rigors were the most common malaria symptoms in adults. ${ }^{6}$ Further, a fact that we all forget is that the Health Protection Agency advises us to consider malaria in a patient who has been potentially exposed up to 12 months. Finally, if the clinical suspicion for malaria is high, three sets of malarial blood films must be taken before suggesting a negative result-easily forgotten by clinicians.

In conclusion, we would like to reinforce the advice given to all clinicians by the Health Protection Agency: it is important to consider malaria due to P. falciparum as a differential in those who have had malaria exposure in the last 12 months and to always take three blood films if there is any clinical suspicion to make a diagnosis of malaria.

Competing interests None

\section{REFERENCES}

1 Reyburn H, Mbatia R, Drakeley C, et .Association of transmission intensity and age with clinical manifestations and case fatality of severe P. falciparum malaria. J Am Med Assoc 2005;293:1461-70

2 Palmer CJ, Lindo JF, Klaskala WI, et al. Evaluation of the OptiMAL test for rapid diagnosis of plasmodium vivax and P. falciparum malaria. J Clin Microbiol 1998;Jan:203-6

3 Kolaczinski J, Mohammed N, Ali I, Ali M, Khan N, Ezard N, Rowland M. Comparison of the OptiMAL rapid antigen test with field microscopy for the detection of Plasmodium vivax and $P$. falciparum: considerations for the application of the rapid test in Afghanistan. Ann Trop Med Parasitol 2004;98:15-20

4 Marx A, Pewsner D, Egger M, et al.Meta-analysis: accuracy of rapid tests for malaria in travellers returning from endemic areas. Ann Internal Med 2005; 142:836-46

5 D'Acremont V, Landry P, Mueller I, Pecoud A, Genton B. Clinical and laboratory predictors of imported malaria in an outpatient setting: an aid to medical decision making in returning travellers with fever. Am J Trop Med Hyg 2002,66:481-6

6 Harrison NE, Odunukwe NN, Ijoma CK, Mafe AG. Current clinical presentation of malaria in Enugu, Nigeria. Niger Postgrad Med J. 2004; 11:240-5 